\title{
Thermal analysis of externally pressurised step bearing including centrifugal inertia effect for a bubbly lubricant
}

\author{
S. S. Gautam ${ }^{1 *}$, S. Quamar ${ }^{2}$, M. K. Ghosh ${ }^{2}$ \\ ${ }^{1 *}$ Department of Mechanical Engineering, North Eastern Regional Institute of Science and Technology, INDIA \\ ${ }^{2}$ Department of Mechanical Engineering, Banaras Hindu University, INDIA \\ *Corresponding Author: e-mail: satyam.gautam@gmail.com,Tel +91-360-2258527, Fax.+91-360-2258533
}

\begin{abstract}
A numerical study has been done for externally pressurized circular step thrust bearing lubricated with incompressible fluid with finely dispersed air bubbles taking into consideration the variation in lubricant physical properties due to temperature and pressure variation in the fluid film. Effect of misalignment / tilt and coning on cavitations region has also been investigated. The air bubbles are seen to improve the pressure distribution. It also reduces cavitations domain that occurs because of the centrifugal inertia force. Load carrying capacity is seen to improve but both mass flow rate and frictional power consumed decrease. Overall, the presence of air bubble improves the performance of the bearing. It is seen that tilt and coning reduces the cavitations region.
\end{abstract}

Keywords: thermal analysis, step bearing, bubbly lubricant, misalignment, coning, cavitations

\section{Introduction}

Circular step hydrostatic thrust bearings and mechanical face seals have similar geometries. However, both have different functions to perform. Thrust bearings are used as load carrying element whereas seals are basically meant to prevent leakage. Externally pressurized bearings where fluid is supplied from an external source can meet some extreme requirement, such as very low frictional characteristics, heavy load at low speed and high positional accuracy. Generally bearing performances are evaluated assuming a constant lubricant viscosity and neglecting the effect of rotation. At high speed of rotation the power dissipated through the lubricant becomes greater and the lubricant temperature rises severely and the assumption of constant viscosity no longer holds well. The effect of shaft rotation also has to be considered.

Cavitations may occur in hydrostatic thrust bearings under axisymmetric condition of operation due to fluid inertia effects. Dowson (1961) and Osterele et. al. (1961) investigated influence of fluid inertia on the performance of hydrostatic thrust bearing and inertia-induced cavitations. Cavitations zone was determined by using Swift-Steiber boundary condition. Dowson introduced the effect of bearing rotation on bearing performance for isoviscous lubricants. He showed that rotational inertia could increase or decrease the bearing load carrying capacity depending upon bearing dimension. Coombs and Dowson (1964) conducted experiments and the results have shown close agreement between the Dowson's theory and their experimental results, especially at low speed. The discrepancy increased with the bearing speed, and they argued that this was caused by neglect of thermal effect in the theoretical analysis. However, they noticed that a negative pressure occurred near the bearing outer periphery. At high speed of rotation the power dissipated through the lubricant becomes greater and lubricant temperature rises severely. Non-axisymmetric condition of operation, which occurs either due to misalignment or provision of coning, hydrodynamic wedge action comes into play when the shaft is rotating. This makes the pressure distribution highly non-axisymmetric and thereby the bearing develops necessary resisting moments. Under non-axisymmetric condition, cavitations may occur due to wedge action generated by shaft rotation. Safar (1980) worked on the performance of circular step hydrostatic thrust bearing under non-axisymmetric condition of operation by assuming full film condition and did not investigate on the cavitations effect.

Many researchers have reported the importance of air or gas/vapor bubbles in a lubricant. It was suggested by Tonder (1975) that the presence of gas bubble might cause a pressure build up. However, the ability of the bubbles to enhance the pressure generating 
capacity of the bearing was shown by him. Tonder (1976) has shown in his paper that with bubbles, the pressure in the region beyond the maximum is seen to drop much more slowly than in the non-bubbly case. The maximum value itself is hardly altered. The load carrying capacity increases markedly with bubbles. Khalil and Rhodes (1980) made a numerical study for externally pressurized bearing lubricated with incompressible fluid and finely dispersed air bubbles, taking into consideration the variation of lubricant physical properties due to pressure and temperature variation along the flow. As the temperature increases from leading edge to trailing edge, the density and viscosity of the lubricant also changes along the radial direction and so their gradient changes. Fenf and Hahn (1986) derived an expression for mixture density which is a function of pressure. There are number of models available in the literature on the viscosity and density of oil-air mixture.

The effects of centrifugal force on the operation of thrust bearings are considered mostly with reference to bearing tapered in angular direction. Such tapers produce film thickness variation both in radial and circumferential direction. Ting and Mayer (1971) have presented a theoretical study for rotational and temperature effects under adiabatic flow condition. This theory was satisfactory as long as no cavitations occurred along the lubricant film. However, cavitations are to be expected at high rotational speed in region of high temperature and low pressure near the bearing's outer periphery. Chen and Dareing (1976) determined contribution of fluid film inertia to thermo-hydrodynamic lubrication of sector-pad thrust bearing. However, cavitations due to centrifugal effect were not looked into. Pinkus and Lund (1981) conducted a thermal analysis to determine centrifugal effects in thrust bearing and seals in laminar flow and found that the centrifugal forces always produce sub-ambient pressure in parallel surfaces. But in tapered thrust bearing they do not always do so. Centrifugal effect can result in large areas of thrust bearings and seals to be deprived of lubricant leading to loss of load capacity. However, in both analyses the lubricant temperature and properties were assumed to vary only over the film thickness i.e. a two dimensional thermo hydrodynamic analysis was presented. Kim et. al. (1983) presented a three dimensional thermo hydrodynamic analysis of a sector shaped tilting pad thrust bearing and evaluated its steady state performance. Abdel-Latif et al. (1985) did a thermo hydrodynamic analysis of thrust bearing with circular pads running on bubbly oil. Qi et al. (1997) presented a study on viscosity properties of bubbly oil and characteristics of journal bearing lubricated with bubbly oil. Literature review show significant recent interest amongst researchers to investigate effect of oil aeration on the performance of journal bearings. Effect of aerated oil on the load capacity of plain journal bearings was studied by Nikolajsen (1999). Nikolajsen also developed models for density and viscosity of aerated oil for use in hydrodynamic lubrication problems. El-Butch (2001) investigated on the influence of bubbly oil on the performance of tilting pad journal bearings under rotating unbalance load. A parametric study on bubbly lubrication of high speed journal bearings has been done by Chun (2002). He has examined the influence of aerated oil on high speed journal bearings by classical thermo hydrodynamic lubrication theory coupled with analytical models for viscosity and density of the air-oil mixture. Both theoretical and experimental investigation was carried out by Goodwin et al. (2007) on the effect of oil aeration on load capacity of journal bearings. Most literature cited deal with analysis of journal bearings running on bubbly oil.

Although air or gas bubbles are often present in the lubricant oil, little seems to have been published on the effect of such bubbles on the performances of hydrostatic thrust bearings. A prevailing assumption in this field seems to be that the presence of gas bubbles is undesirable. An accepted theory of the effect of bubbly lubricants on hydrostatic bearings predicted an improvement of the performance for many types of common bearing design. It was shown that the effect could be explained on the basis of density wedges near the leading and trailing edges of the bearing due to pressure dependency of the density of bubbly lubricants. However, adequate research papers are not available that deal with effects of misalignment / tilt and coning on the performance of circular step hydrostatic thrust bearings operating with bubbly lubricant including thermal and centrifugal effects. Besides, under these situations the extent of cavitations domain has also not been adequately dealt with. This aspect is vital to both circular step hydrostatic thrust bearings and seals.

An analysis of the performance of externally pressurized circular step bearing taking into account the presence of finely disperse air bubbles and the variation in lubricating properties due to variation in the pressure and temperature along the flow has been done. Another aspect is that of cavitations which are expected at high rotational speed in the region of higher temperature and lower pressure near the bearing outer periphery has also been studied. In the present study cavitations region has been found to exist at the outer periphery of bearing land area. Centrifugal effect and the air bubble content influence the cavitations region and the bearing performance as well.

\section{Governing equations}

A typical externally pressurized circular step bearing configuration is shown in the Figure 1. The usual hydrodynamic equations are applied to the flow through the bearing gap taking into the effect of air bubbles on fluid density and viscosity. In this case the film thickness is kept constant along radial as well as circumferential direction. The Reynolds equation is solved for pressure distribution. To determine the temperature distribution energy equation is solved. Another model is that of a hydrostatic thrust bearing having some misalignment with a particular value of coning. The configuration of the misaligned bearing having coning is shown in Figure 2. 


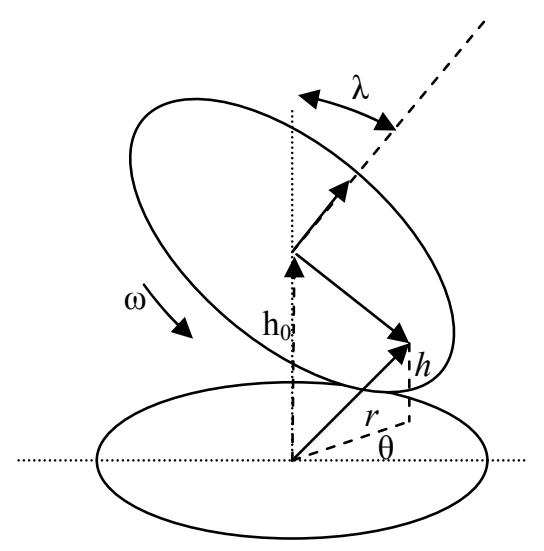

Figure 1: Coordinate system of tilted circular step hydrostatic thrust bearing

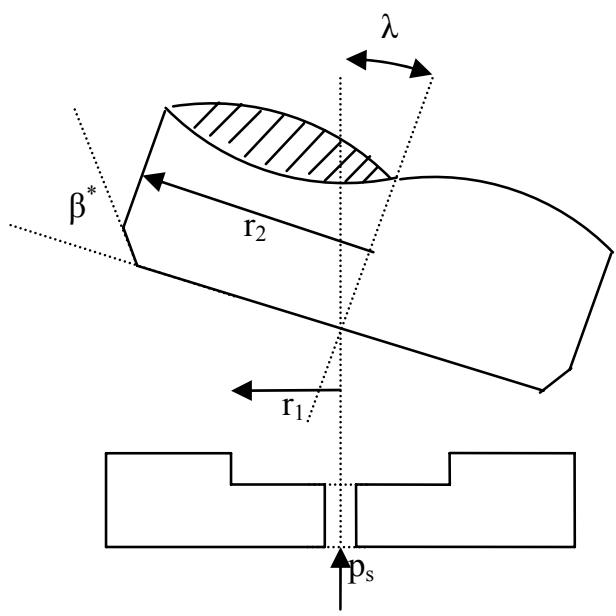

Figure 2: Tilted circular step hydrostatic thrust bearing provided with coning

Influence of presence of air bubbles in the lubricant has been investigated considering centrifugal inertia effect on the performance of hydrostatic thrust bearing. The important manifestation of high rotational speed is that cavitations takes place which reduces the film domain and it becomes severe when centrifugal effect is taken into consideration. It occurs in the region of low pressure and high temperature near the outer periphery. Therefore the theory dealing with hydrostatic step thrust bearings including centrifugal inertia must also involve thermal analysis. Thus, Reynolds equation and energy equation have to be solved simultaneously.

Thus retaining only the centrifugal inertia terms in Navier-Stokes equations, the momentum and continuity equations can be written following Pinkus and Lund (1981) as:

Momentum equations:

$$
\begin{gathered}
\eta \frac{\partial^{2} v_{\theta}}{\partial z^{2}}=\frac{1}{r}\left(\frac{\partial p}{\partial \theta}\right) \\
\eta \frac{\partial^{2} v_{r}}{\partial z^{2}}=\left(\frac{\partial p}{\partial r}\right)-\rho \frac{v_{\theta}^{2}}{r}
\end{gathered}
$$

Continuity equation:

$$
\frac{\partial v_{\theta}}{\partial \theta}+\frac{\partial\left(r v_{r}\right)}{\partial r}+r \frac{\partial v_{z}}{\partial z}=0
$$


Boundary conditions for equations (1) and (2) are given as:

$$
\begin{cases}v_{r}=0, v_{\theta}=r \omega \text { at } z=0, & \text { at the runner surface } \\ v_{r}=0, v_{\theta}=0 \text { at } z=h & \text { at the bearing surface } \\ v_{z}=\frac{\partial h}{\partial t} \text { at } z=0 \& v_{z}=0 \text { at } z=h & \end{cases}
$$

Integration of equations (1) and (2) twice with respect to $\mathrm{z}$ and using the above boundary conditions yields:

$$
\begin{aligned}
& v_{\theta}=\omega(h-z) \frac{r}{h}-\frac{z(h-z)}{2 \eta}\left(\frac{\partial p}{\partial \theta}\right) \\
& v_{r}=\left\{\frac{\rho r \omega^{2}-\frac{\partial p}{\partial r}}{2 \eta}\right\} z(h-z)+\frac{\rho z}{\eta r}\left[A_{1}\left(h^{2}-z^{2}\right)+A_{2}\left(h^{3}-z^{3}\right)+A_{3}\left(h^{4}-z^{4}\right)+A_{4}\left(h^{5}-z^{5}\right)\right]
\end{aligned}
$$

where,

$$
\begin{gathered}
A_{1}=-\frac{\omega}{3 h}\left[\left(\frac{\partial p}{\partial \theta}\right) \frac{h^{2}}{2 \eta}+r^{2} \omega\right] \\
A_{2}=\frac{1}{12}\left\{\frac{r^{2} \omega^{2}}{h^{2}}+\frac{1}{2 \eta}\left(\frac{\partial p}{\partial \theta}\right) \cdot\left[\frac{1}{2 \eta}\left(\frac{\partial p}{\partial \theta}\right) \frac{h^{2}}{r^{2}}+4 \omega\right]\right\} \\
\mathrm{A}_{3}=-\frac{(\partial p / \partial \theta)}{20 \eta}\left[\frac{\left(\frac{\partial p}{\partial \theta}\right) h}{2 \eta r^{2}}+\frac{\omega}{h}\right] \\
\mathrm{A}_{4}=\frac{1}{120} \frac{(\partial p / \partial \theta)^{2}}{\eta^{2} r^{2}}
\end{gathered}
$$

Flow continuity equation (3) can be written in the integral form by integrating across the film thicknesses as:

$$
\int_{0}^{h} \frac{\partial}{\partial \theta}\left(v_{\theta} d z\right)+\int_{o}^{h} \frac{\partial}{\partial r}\left(r v_{r} d z\right)+\int_{o}^{h} r \frac{\partial v_{z}}{\partial z} d z=0
$$

using Liebnitz's rule for integration it becomes:

$$
\begin{gathered}
\frac{\partial}{\partial \theta} \int_{o}^{h} v_{\theta} d z+\frac{\partial}{\partial r} \int_{o}^{h} \frac{\partial}{\partial r} r v_{r} d z-v_{\theta}(h, \theta) \frac{\partial h}{\partial \theta}-v_{r}(h, r) \frac{\partial h}{\partial r}-r v_{z}=0 \\
\text { where } v_{z}=\frac{\partial h}{\partial t}
\end{gathered}
$$

Combining equation (5), (6) and (8) modified Reynolds equation including centrifugal inertia effect of the fluid is obtained as: 


$$
\begin{aligned}
& \frac{\partial}{\partial r}\left\{\frac{r h^{3}}{\eta}\left(\frac{\partial p}{\partial r}\right)\right\}+\frac{1}{r} \frac{\partial}{\partial \theta}\left\{\frac{h^{3}}{\eta}\left(\frac{\partial p}{\partial \theta}\right)\right\}=6 r \omega \frac{\partial h}{\partial \theta}+0.3 \rho \omega^{2} \frac{\partial}{\partial r}\left(\frac{r^{2} h^{3}}{\eta}\right)- \\
& \frac{\rho \omega}{10} \frac{\partial}{\partial r}\left\{\frac{h^{5}}{\eta^{2}}\left(\frac{\partial p}{\partial \theta}\right)\right\}+\frac{3 \rho}{280} \frac{\partial}{\partial r}\left\{\frac{h^{7}}{\eta^{3} r^{2}}\left(\frac{\partial p}{\partial \theta}\right)^{2}\right\}
\end{aligned}
$$

and the corresponding energy equation is written as:

$$
\left(q_{\theta} \frac{\partial T}{\partial \theta}+q_{r} \frac{\partial T}{\partial r}\right)=\frac{\eta}{\rho C_{p}}\left\{\left(\frac{\partial v_{r}}{\partial z}\right)^{2}+\left(\frac{\partial v_{\theta}}{\partial z}\right)^{2}\right\}
$$

where $q_{\theta}$ and $q_{r}$ are lubricant volume flow rates in ' $\theta$ ' and ' $r$ 'directions which can be given by:

$$
\begin{aligned}
& q_{\theta}=\int_{o}^{h} v_{\theta} d z \\
& q_{r}=\int_{o}^{h} v_{r} d z
\end{aligned}
$$

Substituting $v_{\theta} \& v_{r}$ given by equations (5) and (6) and integrating the above expressions, $q_{\theta}$ and $q_{r}$ are obtained as:

$$
\begin{gathered}
q_{\theta}=\frac{r h \omega}{2}-\frac{h^{3}}{12 \eta}\left(\frac{\partial p}{\partial \theta}\right) \\
q_{r}=\frac{h^{3}}{12 \eta}\left[-\left(\frac{\partial p}{\partial r}\right)+0.3 \rho r \omega^{2}+\left\{\frac{3}{280} \frac{\rho h^{4}}{r^{3} \eta^{2}}\left(\frac{\partial p}{\partial \theta}\right)^{2}-\frac{1}{10} \frac{\rho h^{2} \omega}{r \eta}\left(\frac{\partial p}{\partial \theta}\right)\right\}\right]
\end{gathered}
$$

Neglecting pressure and temperature effects the viscosity and density of a bubbly lubricant are given by following expressions (Feng and Hahn, 1986):

$$
\begin{aligned}
& \frac{1}{\eta}=\frac{x}{\eta_{a}}+\frac{1-x}{\eta_{l}} \\
& \frac{1}{\rho}=\frac{x}{\rho_{a}}+\frac{1-x}{\rho_{l}}
\end{aligned}
$$

where $x$ is volume fraction of air. To investigate the influence of centrifugal inertia effects equation (9) and (10) have to be solved using available numerical procedures e.g. finite difference method satisfying proper boundary conditions.

The misaligned or tilted bearing configurations without coning and with coning are shown in Figures 1 and 2. The lubricant film thickness expression can be written as follows:

$$
h=h_{0}+\lambda r \cos \theta+\beta^{*}\left(r-r_{i}\right)
$$

Air viscosity and density are functions of temperature and pressure which can be written as (Khalil and Rhodes, 1980):

$$
\rho_{a}=\frac{p}{R T}, \quad \eta_{a}=\eta_{i a}\left[1+0.00275 T-\left(4.75 * 10^{-7}\right) T^{2}\right]
$$

Lubricant density and viscosity are dependent on temperature only in following manner as given below:

$$
\eta_{l}=\eta_{i l} \exp \left\{\beta\left(T_{i}-T\right)\right\}, \quad \rho_{l}=\rho_{i l}\left\{1+\alpha\left(T_{i}-T\right)\right\}
$$


Boundary conditions used are expressed as:

$$
\begin{cases}p=p_{s} & \text { at } r=r_{1} \\ p=0 & \text { at } r=r_{2} \\ p=\frac{d p}{d \theta}=\frac{d p}{d r}=0, & \text { at the cavitation boundary } \\ T=T_{i} & \text { at } r=r_{1}\end{cases}
$$

Load capacity, frictional power loss and mass flow rate are determined for known pressure distribution. Thus,

$$
\text { Load capacity, } \quad W=\int_{0}^{2 \pi} \int_{r_{1}}^{r_{2}} p r d r d \theta
$$

Frictional power loss factor is determined from frictional torque as:

$$
P_{f}=\int_{0}^{2 \pi} \int_{r_{1}}^{r_{2}} \omega \tau_{r \theta} r^{2} d r d \theta=\int_{0}^{2 \pi} \int_{r_{1}}^{r_{2}} \eta \frac{r \omega^{2}}{h} r^{2} d r d \theta
$$

Lubricant mass flow rate is determined as:

$$
M=\int_{0}^{2 \pi} \int_{0}^{h} \rho v_{r} r d z d \theta
$$

Dimensionless load capacity $\bar{W}$, frictional power loss $\bar{P}_{f}$ and mass flow rate $\bar{M}$ are determined from the following expressions.

$$
\begin{aligned}
& \bar{W}=\frac{W}{p_{s} \pi r_{2}^{2}} ; \\
& \bar{P}_{f}=\frac{P_{f}}{\eta_{i} r_{2}^{3} \omega^{2}}\left(\frac{h_{2}}{r_{2}}\right) ; \\
& \bar{M}=\frac{M}{\rho_{i} L^{2} \omega h_{2}}
\end{aligned}
$$

where $L=\left(r_{2}-r_{1}\right)$ and $h_{2}$ is the minimum film thickness at the outer periphery of the bearing.

\section{Results and discussions}

Performance of misaligned externally pressurized circular step bearing in terms of pressure, temperature, load capacity, power loss and mass flow rate are presented which show the influence of speed of rotation and the air bubble content. It is seen that air bubble content significantly influences the bearing performance.

Reynolds equation for hydrostatic step thrust bearings under axisymmetric condition of operation as given by equation (9) was expressed in finite difference form and then solved for pressure distribution by Gauss-Siedel iteration method satisfying boundary conditions at the recess and the outer periphery given in equation (18). Reynolds boundary for film rupture was satisfied by setting negative pressure equal to zero as and when occurred during the process and iteration proceeded there after. Cavitations regions were thus determined. The process of iteration was terminated at a point where a prescribed convergence criterion was satisfied between two consecutive iterations. Similarly energy equation (10) was also solved using finite difference method iteratively satisfying boundary conditions at the oil inlet radius. The thermal solution considered converged when both Reynolds equation 
and energy equation converged simultaneously. The various input parameters are given in Table A1. The algorithm and flow chart for the solution is given in Appendix.

Figure 3 shows the influence of air bubble content on the pressure distribution. For the journal rotating at a speed of $200 \mathrm{rad} / \mathrm{s}$, it is clear from figure that there is an improvement in pressure profiles as the percentage of air bubble increases. An interesting feature is that at lower percentage of air bubble content between 0.0 and 0.01 pressure distribution is seen to improve significantly, but at high air bubble content i.e. between 0.01 and 0.1 , the effect is not very predominant. For a hydrostatic thrust bearing the effect of centrifugal inertia is to create cavitations zone in the trailing edge of the bearing. At higher speed the centrifugal term is more predominant, and so larger bearing area is seen to be cavitated. In Figure 4 the pressure profiles are shown for three rotational speeds of 500, 200 and $0 \mathrm{rad} / \mathrm{s}$ respectively for bubbly oil with 10 percent air bubble. At $500 \mathrm{rad} / \mathrm{s}$ cavitated area is larger than that of 200 and $0 \mathrm{rad} / \mathrm{s}$ which shows the influence of centrifugal inertia on the film extent. Corresponding temperature distribution is shown in Figure 5. Temperature in the bearing land region increases significantly with increase in rotational speed. This is due significant increase in viscous shear heating at high rotational speeds. The lubricant exits from the bearing periphery at higher temperature. Pressure distributions obtained from both isothermal and thermal analysis is shown in Figure 6 . It is seen that higher pressures are obtained from the thermal analysis.

The radial pressure distribution of an externally pressurized bearing calculated from the present theory is presented without and with the air bubble for different rotation speeds. For a bearing with a recess to outer radius ratio of 0.67 a negative pressure is expected owing to rotation and its domain can vary with bearing speed air bubble content. Presence of air bubble in the lubricant alters the pressure distribution and to some extent prevents the generation of negative pressure, thus can improve the bearing load carrying capacity by increasing the positive pressure domain.

Figures 7 to 12 show the effect of air bubble content on the lubricant mass flow rate, load carrying capacity and frictional power respectively. The lubricant mass flow rate decreases with increase in air bubble content for parallel configuration when film thickness is constant. However, it increases with an increase in speed of the runner due to centrifugal effect. The load carrying capacity always improves with an increase in air bubble content. But this improvement is not very much effective at higher range of air bubble content. At high rotational speeds, the load carrying capacity decreases. Frictional power loss decreases with an increase in air bubble content whereas it increases with increase in angular speed due to increase in viscous shear effect.

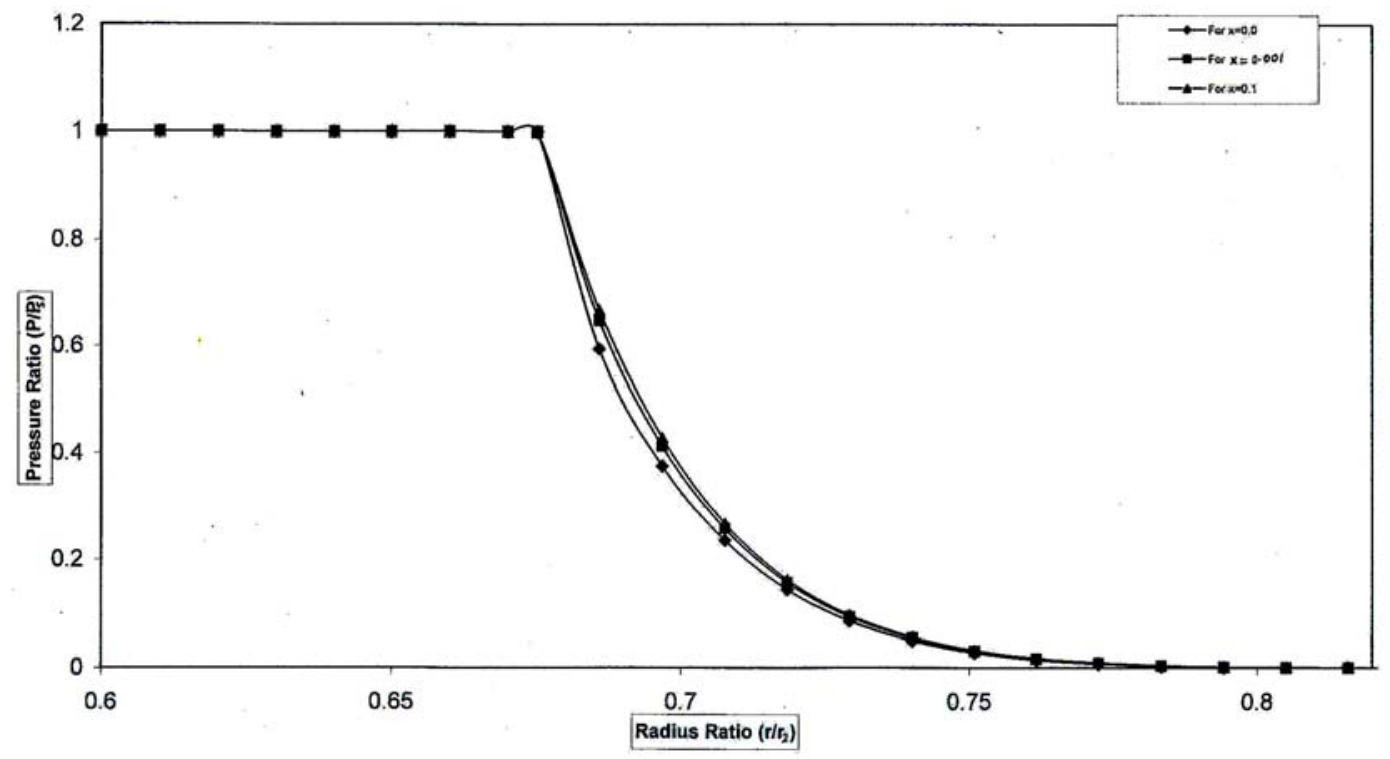

Figure 3: Effect of air bubble on the pressure distribution in circular step hydrostatic bearing at a speed of $200 \mathrm{rad} / \mathrm{s}$ 


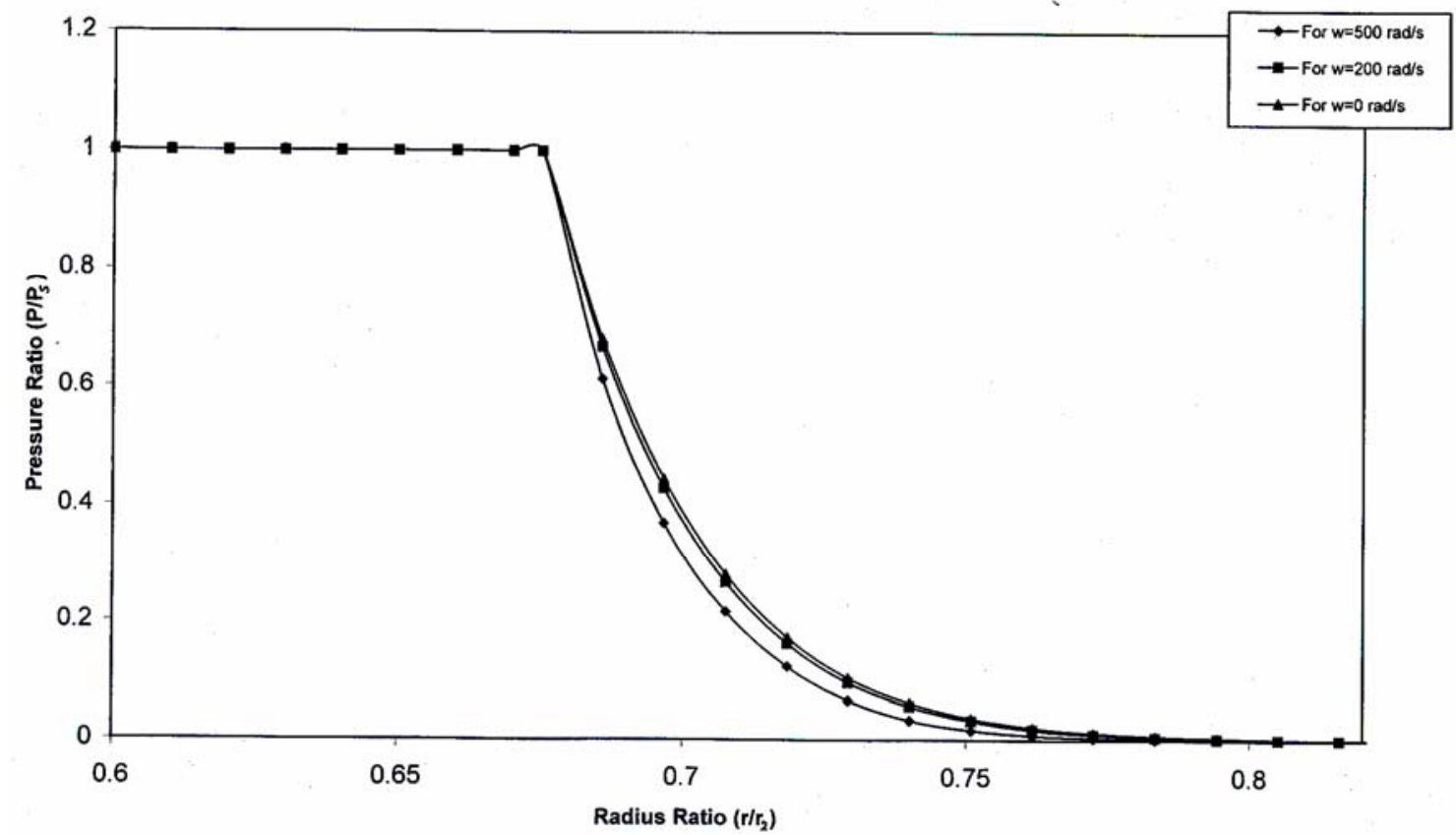

Figure 4: Effect of speed on pressure distribution at $x=0.1$ in circular step hydrostatic thrust bearing

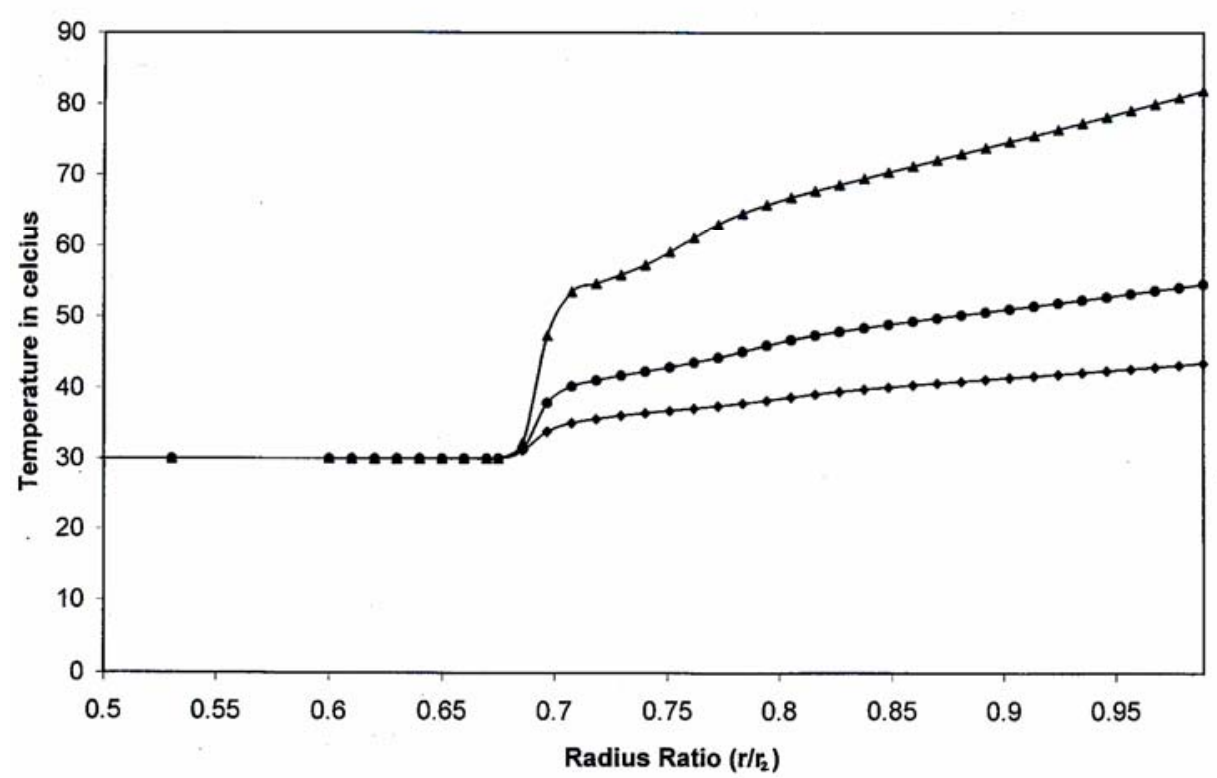

Figure 5: Effect of speed on temperature distribution at $x=0.1$ in circular step hydrostatic bearing 


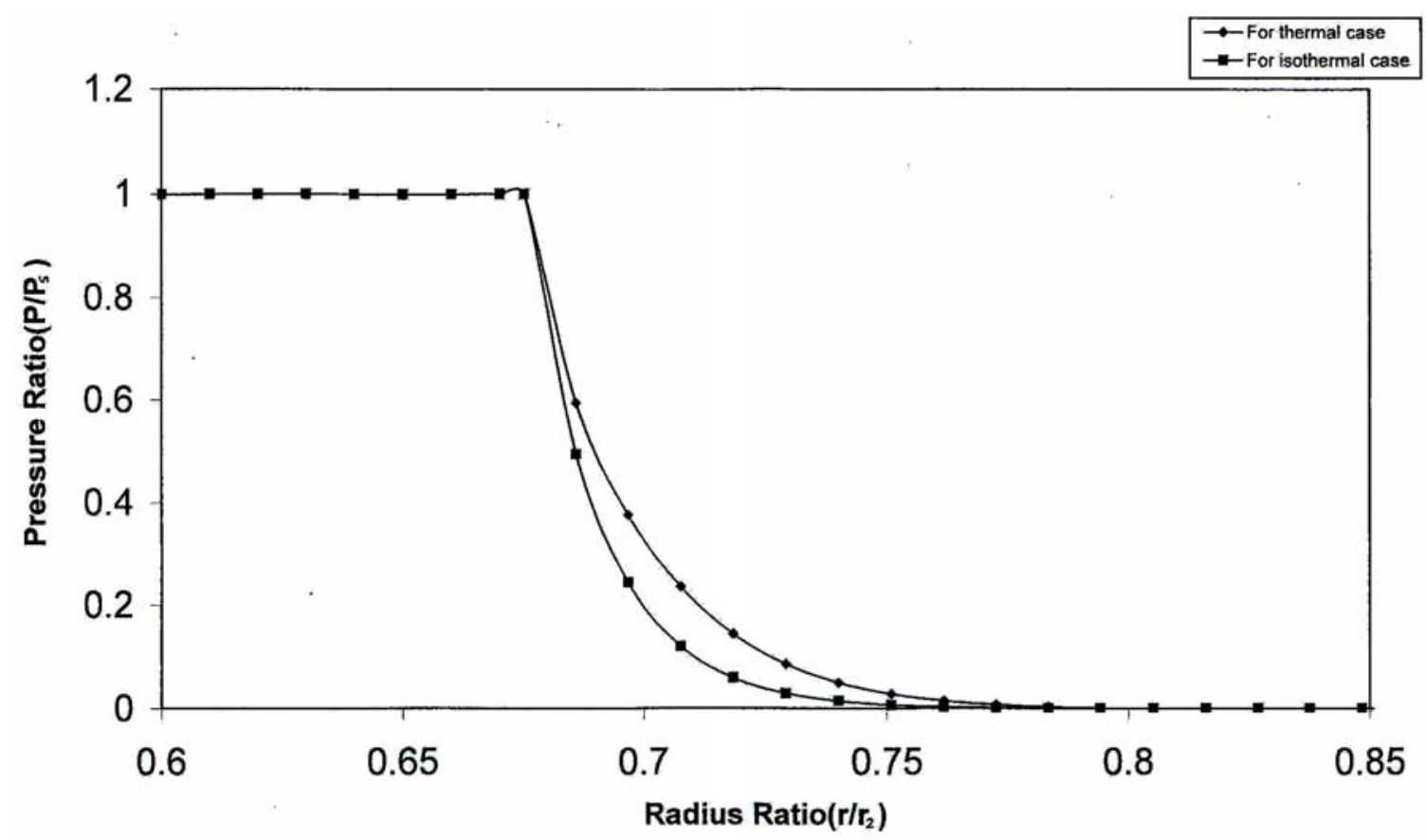

Figure 6: Pressure distribution along radial direction at a speed of $200 \mathrm{rad} / \mathrm{s}$

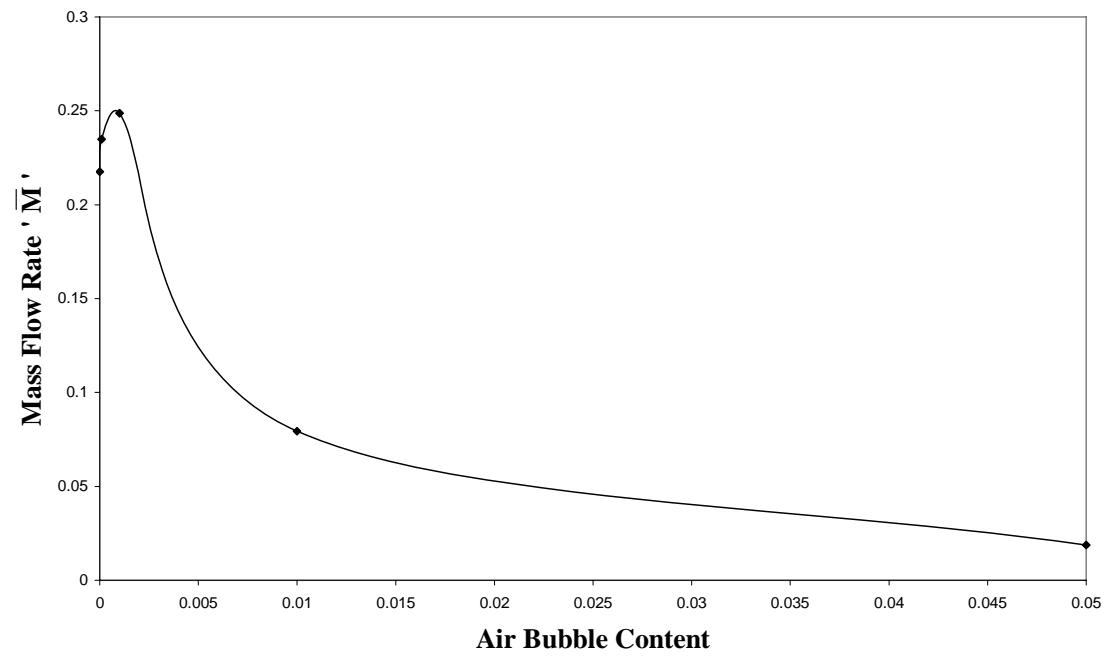

Figure 7: Variation in mass flow rate due to variation in air bubble content 


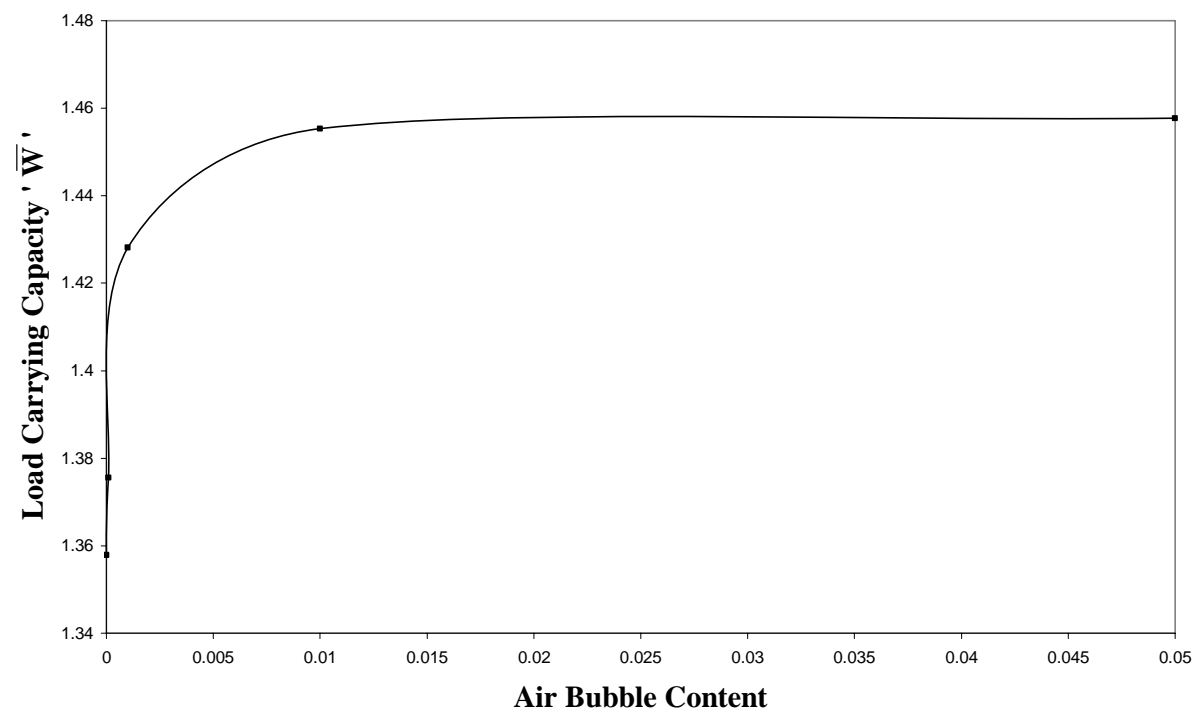

Figure 8: Variation in load carrying capacity due to variation in air bubble content

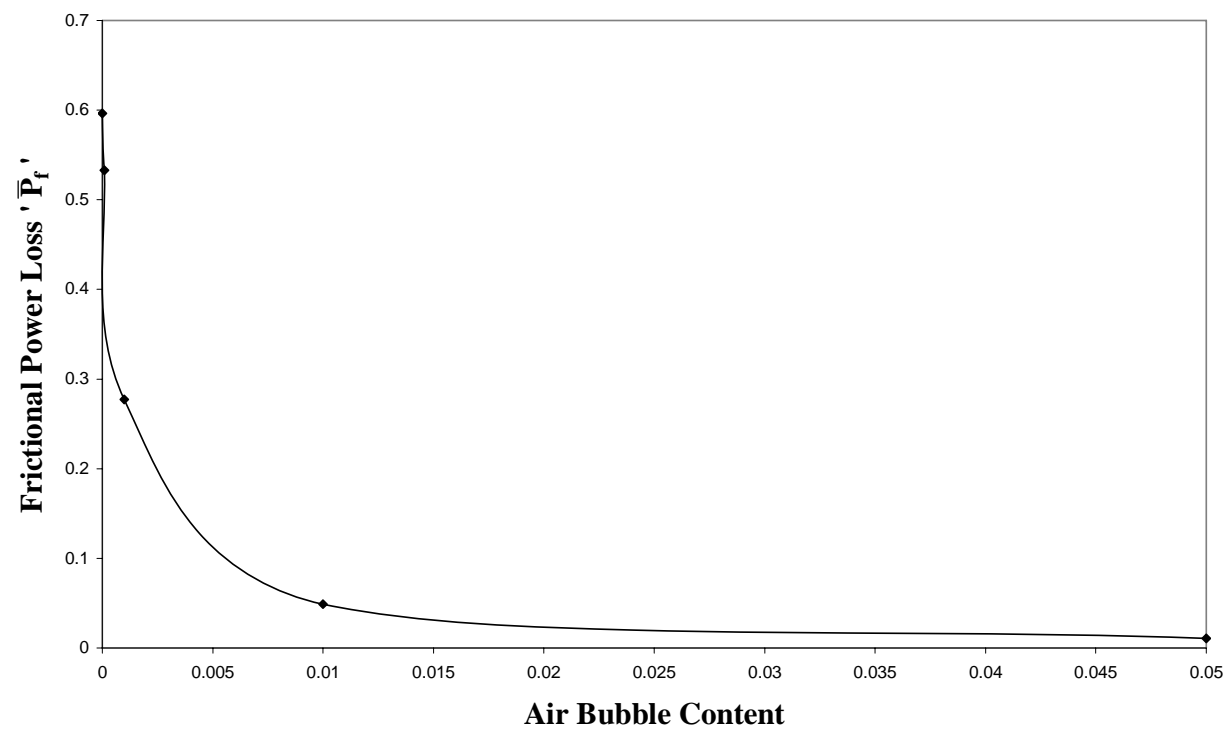

Figure 9: Variation in frictional power loss due to variation in air bubble content 


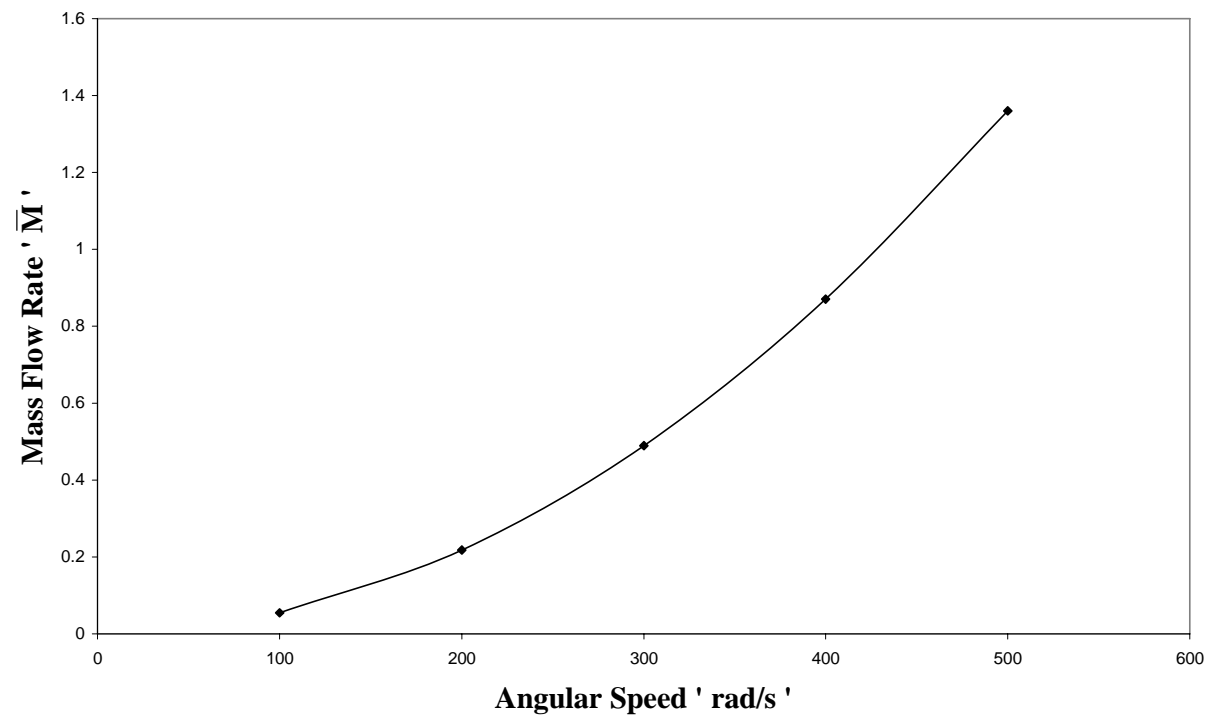

Figure 10: Variation in mass flow rate due to variation in angular speed

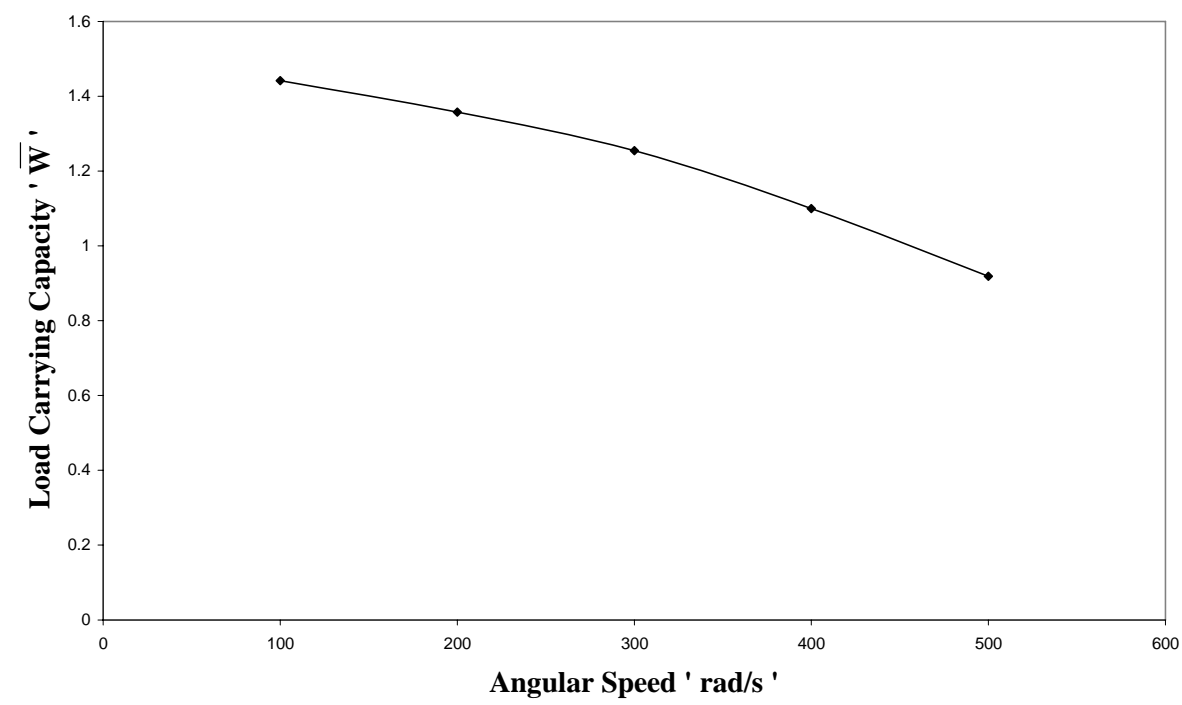

Figure 11: Variation in load carrying capacity due to variation in angular speed 


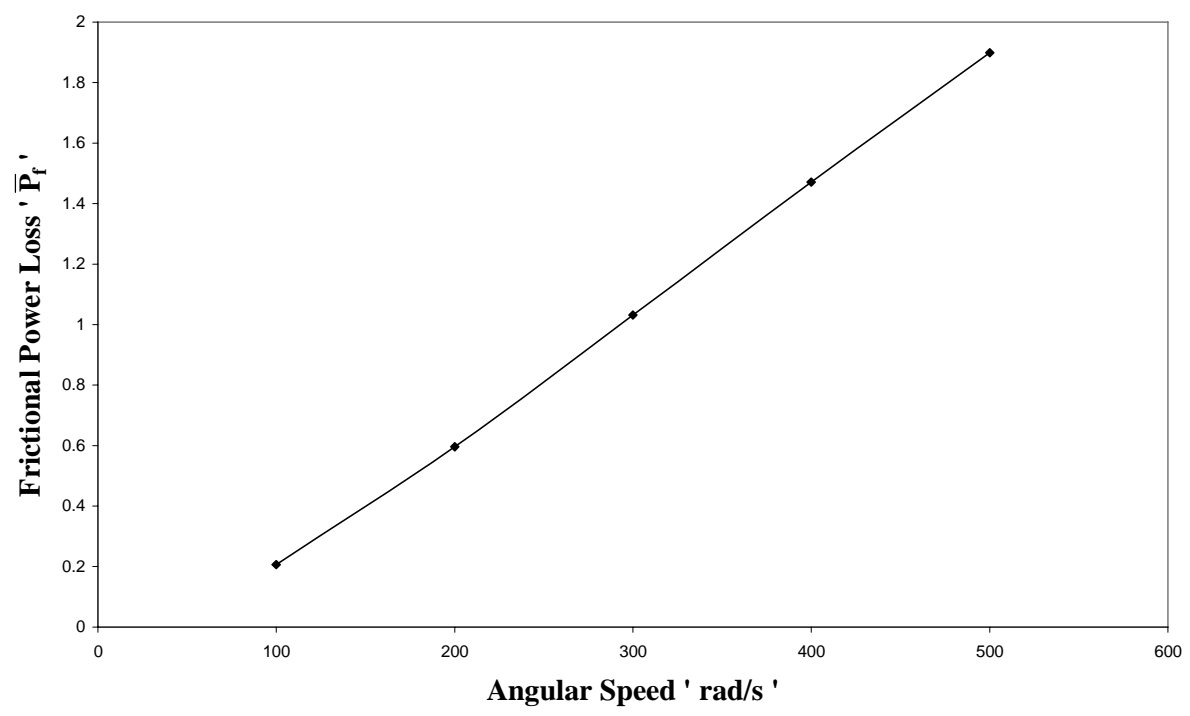

Figure 12: Variation in frictional power loss due to variation in angular speed

Influence of air bubble content on the cavitations zone can be seen in the bearing for both parallel and misaligned configurations. One can clearly see that cavitations zone is reduced with the inclusion of air bubble in the lubricant. Location and size of cavitations zone is shown through Figures 13 to 18. Cavitations occur due to hydrodynamic wedge action under tilted condition. Due to tilt, film thickness becomes convergent-divergent. Pressure increases in the convergent portion of the film and drops in the divergent portion causing cavitations due to wedge action at bearing speed. However, when the shaft is stationary wedge action is absent and cavitation cannot take place. But pressure distribution is still non-symmetric due to tilt. Figures 13 and 14 are for parallel configuration of the bearing. In Figures 15 and 16 cavitation zones are shown for tilted configuration of the bearing at an angular velocity of $100 \mathrm{rad} / \mathrm{s}$ for different amount of air bubble content. The region of cavitations gradually decreases as the amount of air bubble content is increased. Figures 17 and 18 show similar trend in the cavitation region of the bearing provided with coning.

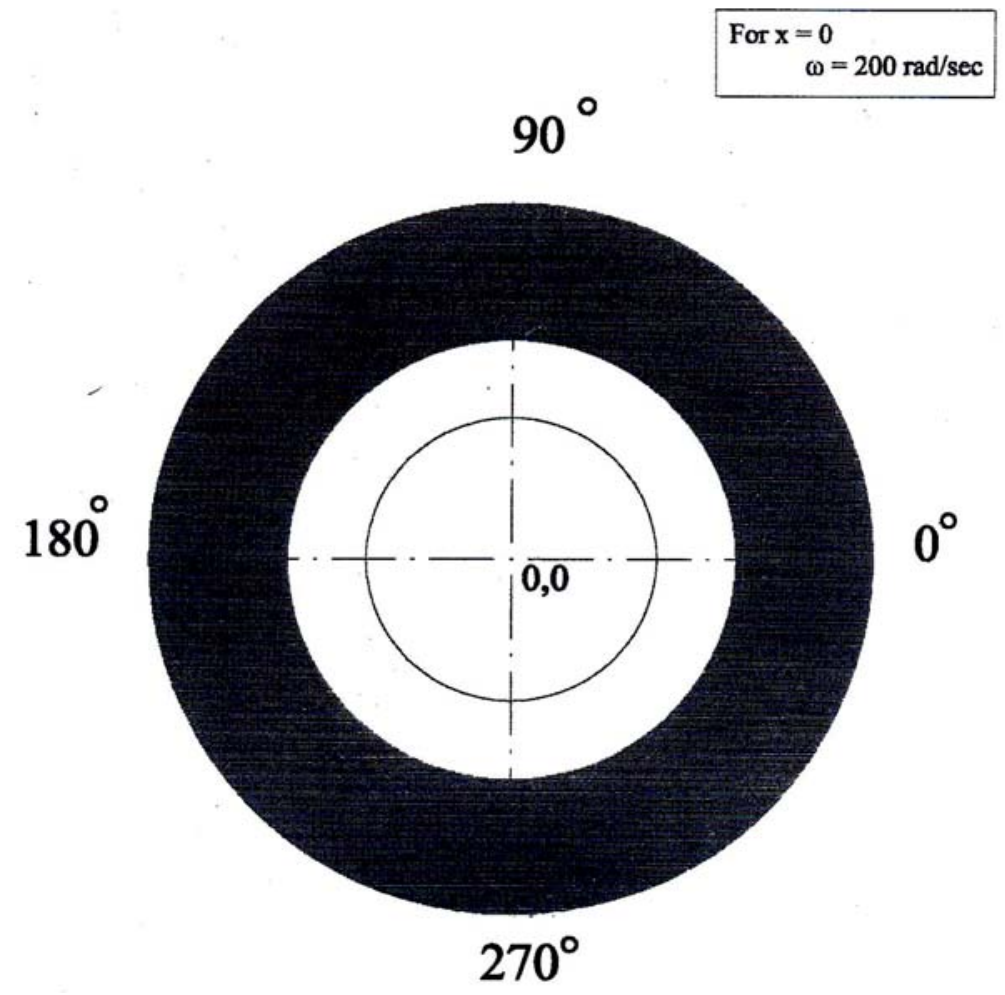

Figure 13: Cavitations zone on bearing land surface in circular step hydrostatic thrust bearing 


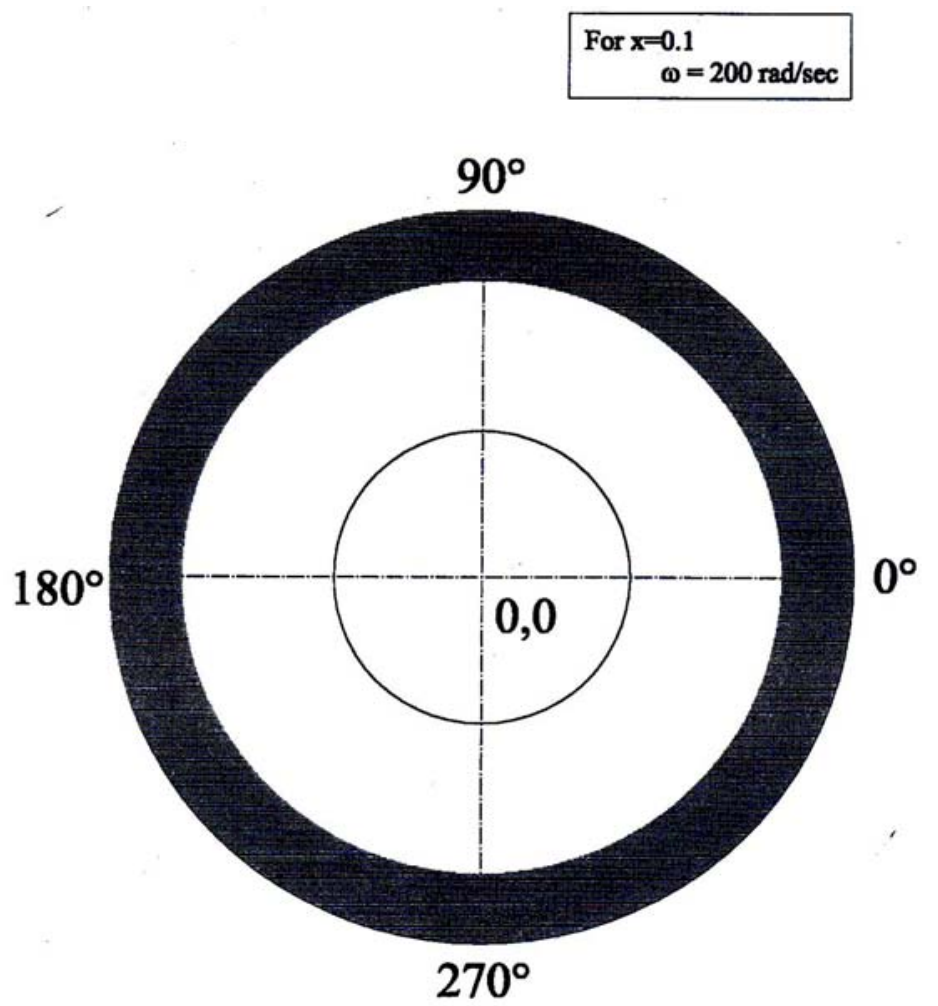

Figure 14: Cavitations zone on bearing land surface in circular step hydrostatic thrust bearing

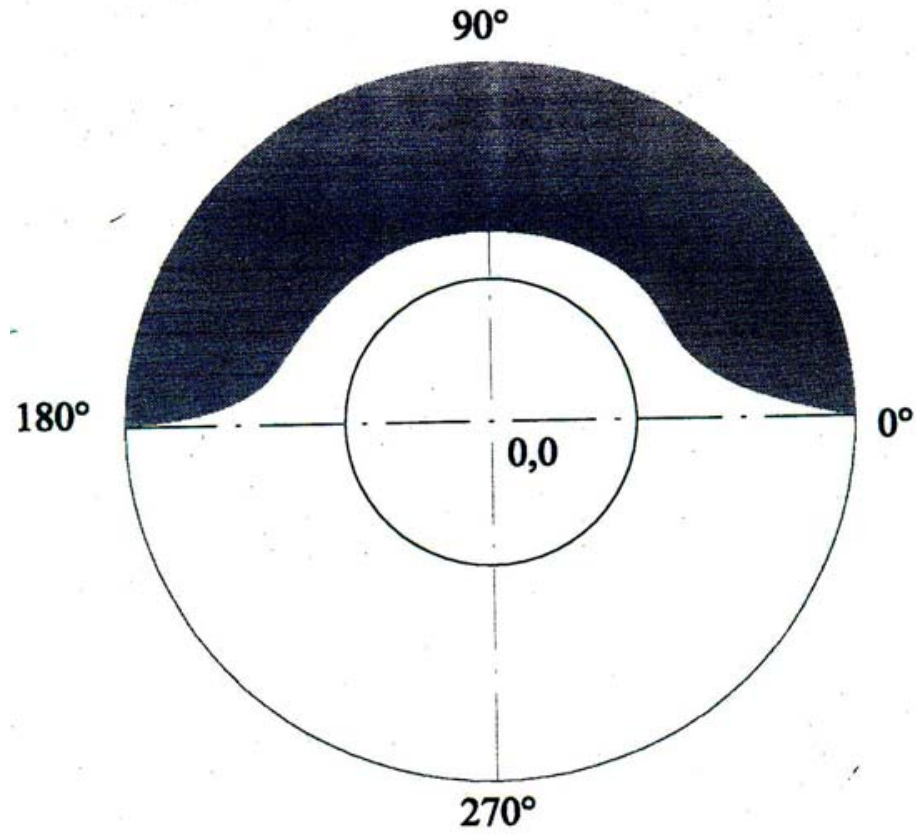

Figure 15: Cavitations zone on bearing land surface in circular step hydrostatic thrust Bearing $(\lambda=0.001265 \mathrm{rad})$ 


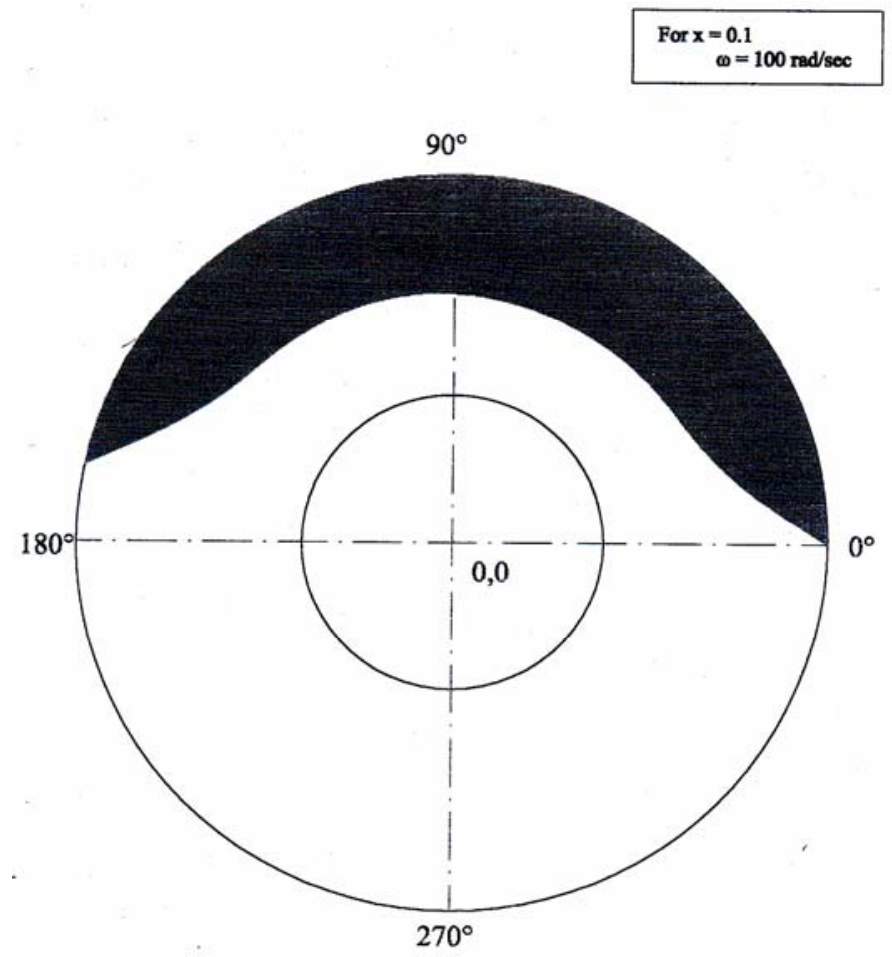

Figure 16: Cavitations zone on bearing land surface in circular step hydrostatic thrust bearing $(\lambda=0.001265 \mathrm{rad})$
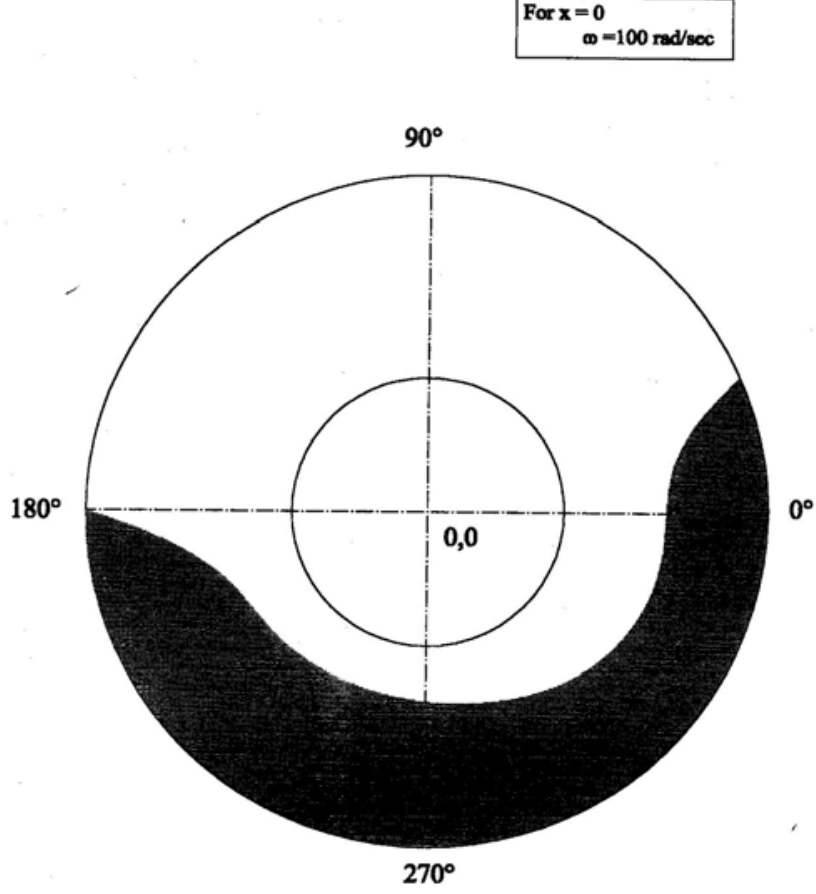

Figure 17: Cavitations zone on bearing land surface in circular step hydrostatic thrust bearing $\left(\beta^{*}=0.004219 \mathrm{rad}\right)$ 


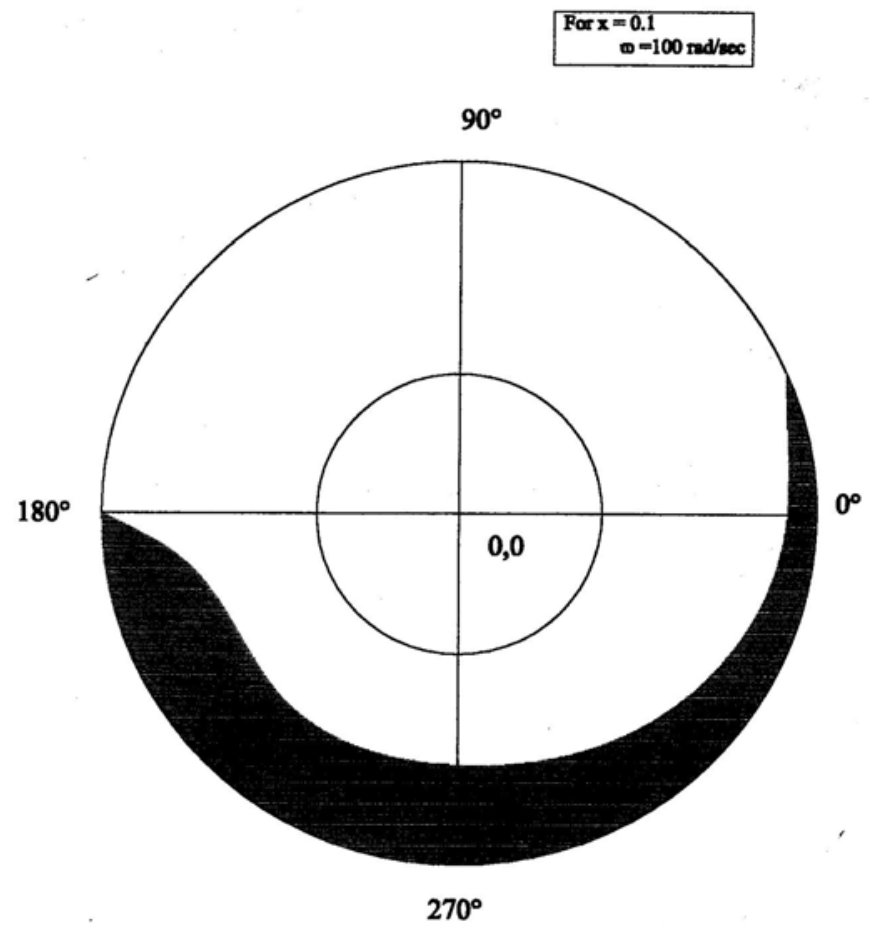

Figure 18: Cavitation zone on bearing land surface in circular step hydrostatic thrust bearing $\left(\beta^{*}=0.004219 \mathrm{rad}\right)$

\section{Conclusions}

The following conclusions can be drawn from the results presented.

1. Bubbly oil improves the performance of the bearing when the air bubble content is low. Higher air bubble content does not alter the performance. There is thus an optimum value of air bubble content in the oil $(x=0.01)$ up to which performance improves.

2. Temperature increases from leading edge to trailing edge. There is a surge in temperature at the recess edge due to discontinuity in the film thickness.

3. Load carrying capacity improves initially with increase in the air bubble content and then asymptotically attains a constant value when air bubble content becomes high.

4. Region of cavitations diminishes with increase in the air bubble content. Due to centrifugal inertia effect cavitations zone increases.

5. The frictional power loss and lubricant mass flow rate decreases with the air bubble content.

6. Cavitations region is reduced by misalignment and coning.

\section{Nomenclature}

$C_{p} \quad$ Specific heat at constant pressure

$h \quad$ Film thickness

$h_{0} \quad$ Film thickness at center

$h_{2} \quad$ Film thickness at the outer periphery of the bearing

$L \quad$ Radial extent of bearing length

$m \quad$ Number of divisions in ' $\theta$ ' direction

$M \quad$ Lubricant mass flow rate, $\bar{M}=\frac{M}{\rho_{i} L^{2} \omega h_{2}}$

$n \quad$ Number of divisions in ' $r$ ' direction

$N \quad$ Speed of rotation

$p \quad$ Pressure 


\begin{tabular}{|c|c|}
\hline$p_{s}$ & Supply pressure \\
\hline$P_{f}$ & Frictional power loss, $\bar{P}_{f}=\frac{P_{f}}{\mu_{i} r_{2}^{3} \omega^{2}}\left(\frac{h_{2}}{r_{2}}\right)$ \\
\hline$q_{\theta}$ & Volume flow rate in ' $\theta$ ' direction \\
\hline$q_{r}$ & Volume flow rate in ' $r$ ' direction \\
\hline$r$ & Axial location \\
\hline$r_{1}$ & Recess radius \\
\hline$r_{2}$ & Outer radius of bearing \\
\hline$R$ & Gas constant of air \\
\hline$T$ & Absolute temperature \\
\hline$T_{i n}$ & Lubricant inlet temperature \\
\hline$v_{\theta}$ & Velocity component in ' $\theta$ ' direction \\
\hline$v_{r}$ & Velocity component in ' $r$ ' direction \\
\hline$v_{Z}$ & Velocity across the film thickness \\
\hline$W$ & Load carrying capacity, $\quad \bar{W}=\frac{W}{}$ \\
\hline$x$ & Mass ratio of air bubbles to mixture \\
\hline$z$ & Radial coordinate \\
\hline$\alpha$ & Coefficient of thermal expansion of lubricant \\
\hline$\beta$ & Coefficient of viscosity \\
\hline$\beta^{*}$ & Coning angle \\
\hline$\eta$ & Viscosity of lubricant \\
\hline$\lambda$ & Angle of misalignment \\
\hline$\omega$ & Angular velocity of rotation \\
\hline$\rho$ & Density of the lubricant \\
\hline$\theta$ & Tangential coordinate \\
\hline$\tau$ & Shear stress \\
\hline
\end{tabular}

Subscripts

$\begin{array}{llll}a & \text { air } & 1 & \text { recess edge } \\ l & \text { liquid } & 2 & \text { outer radius of the bearing } \\ i & \text { inlet } & & \end{array}$

\section{References}

Abdel-Latif L. A., Peeken H., Benner J., 1985. Thermohydrodynamic analysis of thrust bearing with circular pads running on bubbly oil (BTHD-theory). ASME Journal of Tribology, Vol. 107, No. 4, pp. 527-537.

An Q.., Yinsheng Z., Yongxin Q., 1997. Study on the viscosity properties of bubbly oil and the static characteristics of journal bearing lubricated with bubbly oil. Wear, Vol. 213, pp. 159-164.

Chen C. M., Dareing D. W., 1976, The contribution of fluid film inertia to the thermohydrodynamic lubrication of sector-pad thrust bearings. ASME Journal of Lubrication Technology, Vol. 98, pp. 125-132.

Chun S. M., 2002. A parametric study on bubbly lubrication of high speed journal bearings. Tribology International, Vol. 35, pp. $1-13$.

Coombs J. A., Dowson D., 1964. An experimental investigation of the effects of lubricant inertia in a hydrostatic thrust bearing. Proceedings Lubrication and Wear - $3^{\text {rd }}$ Convention, Inst. of Mech. Engrs, London, 179(3J), Paper 12, pp. 96-108.

Dowson D., 1961. Inertia effect in hydrostatic thrust bearing. ASME Journal of Basic Engineering, Vol. 83, Ser. D, pp. 227-234. 
El-Butch A. M. A., 2001. The effect of bubbly oil on the THD lubrication of tilting pad journal bearings subjected to rotating unbalanced load. Meccanica, Vol. 36, pp. 717-729.

Feng N. S., Hahn E. J., 1986. Density and viscosity model for two phase homogeneous hydrodynamic damper fluids. ASLE Transaction, Vol. 29, No. 3, pp. 361-369.

Goodwin M. J., Dong D., Yu H., Nikolajsen J. J., 2007. Theoretical and experimental investigation of the effect of oil aeration on the load carrying capacity of a hydrodynamic journal bearing. Proceedings of IMechE Journal of Engineering Tribology Part J, Vol. 221, pp. 779-786.

Khalil M. F., Rhodes E. 1980. Effect of air bubble on externally pressurised bearing performances. Wear, Vol. 65, pp. 113-123.

Nikolajsen J. J., 1999. The effect of aerated oil on the load capacity of a plain journal bearing. ASLE Tribology Transaction, Vol. 42, No. 1, pp. 58-62.

Kim K. W., Tanaka M., Hori Y., 1983. A three dimensional analysis of thermo hydrodynamic performance of sector shaped tilting pad thrust bearing. ASME Journal of Lubrication Technology, Vol. 105, pp. 406-413.

Nikolajsen J. J., 1999. Viscosity and density models for aerated oil in fluid film bearings. ASLE Tribology Transaction, Vol. 42, No. 1, pp. 186-191.

Osterele J. F., Hughes W. F., 1961. Inertia induced cavitations in hydrostatic thrust bearing. Wear, Vol. 4, pp. $228-233$.

Pinkus O., Lund J. W., 1981. Centrifugal effects in thrust bearings and seals under laminar conditions. ASME Journal of Lubrication Technology, Vol. 103, pp.126-136.

Safar Z. S., 1980. Performance characteristics for the hydrostatic thrust bearing of a saw blade. Tribology International, Vol. 13, No.2, pp. 79-83.

Tonder K., 1975. Parallel Surface Lubricated by Bubbly Oil. Wear, Vol. 35, pp. 23-34.

Tonder K., 1976. Thermal model of effects of gas bubbles on the lubrication of parallel surface. Wear, Vol. 40, No. 1, pp. 37-50.

Ting L. L., Mayer J. E., 1971. The effect of temperature and inertia on hydrostatic thrust bearing performance. ASME Journal of Lubrication Technology, Vol. 93, No. 2, pp. 307-312.

\section{Biographical notes}

Dr. S. S. Gautam received his M. Tech. and Ph.D. degree from Banaras Hindu University in the Department of Mechanical Engineering, Varanasi, India, in 2002 and 2006 respectively. He is presently working as an Assistant Professor in North Eastern Regional Institute of Science and Technology (NERIST) in Mechanical Engineering Dept. since 2006. He is a life member of Indian Society of Technical Education (ISTE) as well as Tribology Society of India (TSI). He has published 6 international research papers and has guided 4 UG project. He has been awarded UGC fellowship during his M. Tech. and Ph.D. degree. His research area is Tribology of bearings and seals.

S. Quamar is with the Department of Mechanical Engineering, Banaras Hindu University, India

Prof. M.K. Ghosh received his B.S. (Mechanical Engineering) in 1966 from Banaras Hindu University, Varanasi, India, M.Tech in 1968 and Ph.D. in 1979 in Mechanical Engineering respectively from Indian Institute of Technology, Kharagpur, India. Professor Ghosh joined as a faculty member in 1969 in the Department of Mechanical Engineering, Institute of Technology, Banaras Hindu University, and Varanasi, India and is a Professor since 1993. He has worked as a NRC Research Associate during 1982-1984 as a senior NRC Research Associate during 1989-90 at the NASA Lewis Research Center, Cleveland, OH (USA). He was a Professor of Mechanical Engineering in the Department of Mechanical Engineering at I.I.T. Kharagpur during 1987-88. Professor Ghosh is a member of the American Society of Mechanical Engineers since 1984, a Life Member of the Tribology Society of India (TSI) and was a member of the Executive Committee of the TSI during 1995-97. He is a member of the honorary editorial board of the journal "Advances in Vibration Engineering", the scientific journal of the "Vibration Institute of India". He is a recipient of the University Grants Commission of India "Career Award" in Engineering in 1984. Prof. Ghosh's research interest areas are: Tribology, Vibration, and Dynamics \& Control of Mechanical Systems. He has published about 45 papers in peer reviewed international journals e.g. Tribology Transactions of ASME, Proc. of I. Mech. E., U.K., Tribology International, Int. J. of Wear, Int. J. of Mechanical Sciences, Int. J. of Machine Tools \& Manufacturing etc. Professor Ghosh has supervised about 35 graduate thesis and $6 \mathrm{Ph} . \mathrm{D}$. students.

Received September 2010

Accepted December 2010

Final acceptance in revised form December 2010 


\section{Appendix}

Table A1: List of input parameters

\begin{tabular}{|l|c|}
\hline \multicolumn{1}{|c|}{ Input data } & Values \\
\hline Recess radius, $r_{l}$ & $0.16 \mathrm{~m}$ \\
\hline Outer radius, $r_{2}$ & $0.237 \mathrm{~m}$ \\
\hline Film clearance, $C$ & $10^{-4} \mathrm{~m}$ \\
\hline Radial extent of bearing, $L$ & $0.077 \mathrm{~m}$ \\
\hline Angular speed, $\omega$ & $0-500 \mathrm{rad} / \mathrm{s}$ \\
\hline Supply pressure, $p_{s}$ & $1.5 * 10^{5} \mathrm{~N} / \mathrm{m}^{2}$ \\
\hline Lubricant inlet temperature, $T_{i}$ & $30^{0} \mathrm{C}$ \\
\hline Lubricant inlet density, $\rho_{\mathrm{i}}$ & $855 \mathrm{~kg} / \mathrm{m}^{3}$ \\
\hline Lubricant inlet viscosity, $\eta_{\mathrm{i}}$ & $5^{*} 10^{-2} \mathrm{Ns} / \mathrm{m}^{2}$ \\
\hline Coefficient of thermal expansion of lubricant, $\alpha$ & $7.34^{*} 10^{-4} \mathrm{C}^{-1}$ \\
\hline Coning angle, $\beta^{*}$ & $0.004219 \mathrm{radian}$ \\
\hline Coefficient of viscosity, $\beta$ & $4.91^{*} 10^{-2}{ }^{0} \mathrm{C}^{-1}$ \\
\hline Angle of misalignment, $\lambda$ & $0.001265 \mathrm{radian}$ \\
\hline Specific heat at constant pressure, $C_{p}$ & $2.02 * 10^{3} \mathrm{~J} / \mathrm{kg}^{0} \mathrm{C}$ \\
\hline Gas constant of air, $R$ & $287 \mathrm{~J} / \mathrm{kg} \cdot \mathrm{mole} /{ }^{0} \mathrm{~K}$ \\
\hline Air viscosity, $\eta_{a}$ & $1.9^{*} 10^{-5} \mathrm{Ns} / \mathrm{m}^{2}$ \\
\hline
\end{tabular}




\section{Flow Chart}

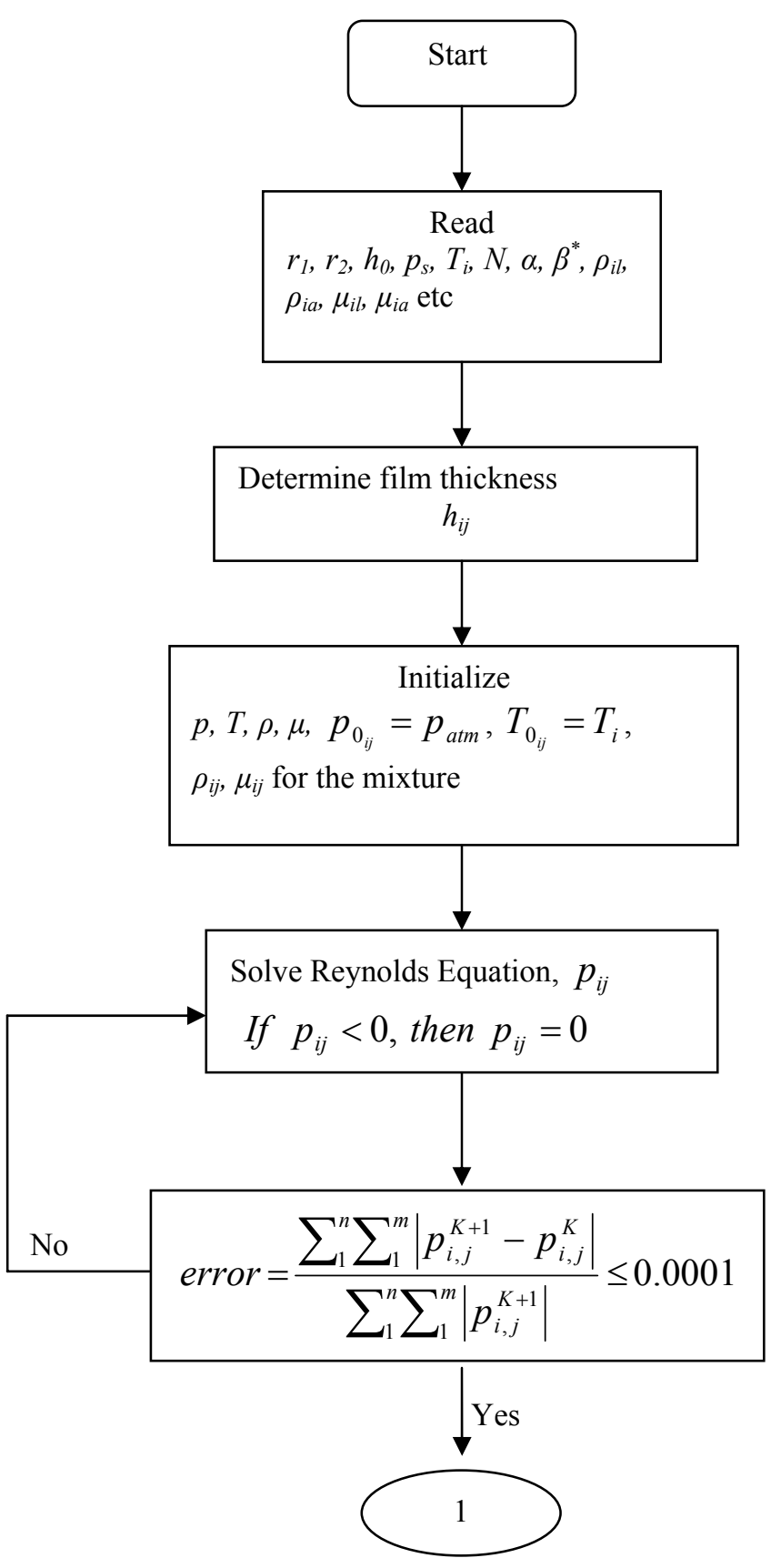




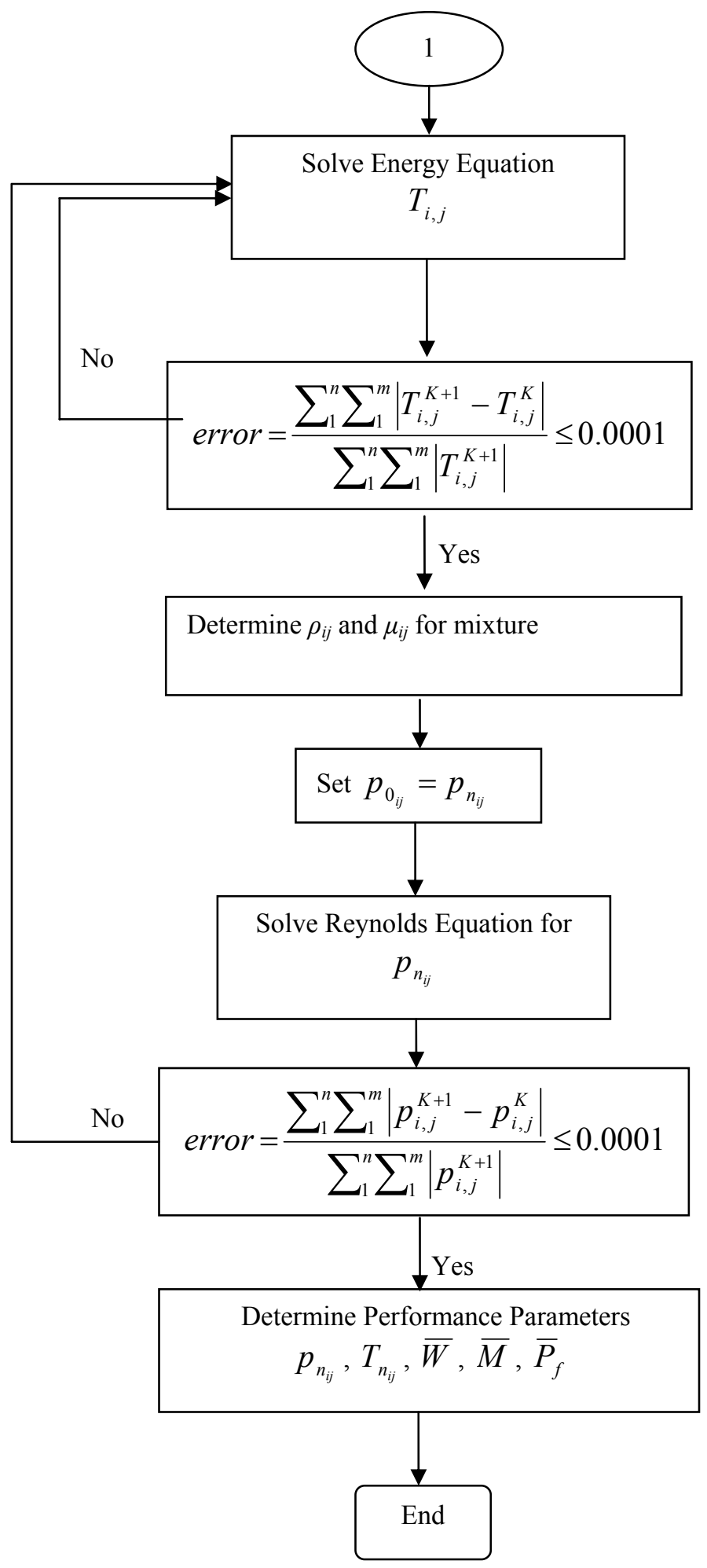

